\title{
Evaluation of Oxygen Uptake Kinetic Asymmetries in Patients with Multiple Sclerosis: A Pilot Study
}

Rebecca D. Larson'*, Monica Barton ${ }^{1}$, John W Farrell III' ${ }^{1,2}$, Gregory S. Cantrell ${ }^{1,3}$, David J. Lantis ${ }^{1,4}$, Christopher D. Black ${ }^{1}$, Carl J. Ade ${ }^{1,5}$

${ }^{I}$ Department of Health and Exercise Science, University of Oklahoma, Norman, OK, USA

${ }^{2}$ Interdisciplinary School of Health Sciences, University of Ottawa, Ottawa, ON, CDN.

${ }^{3}$ Health and Physical Education Department, Northern State University, Aberdeen, SA, USA

${ }^{4}$ Department of Kinesiology, St. Ambrose University, Davenport, IA, USA

${ }^{5}$ Department of Kinesiology, Kansas State University, Manhattan KS, USA

Corresponding Author: Rebecca D Larson, E-mail: rdlarson@ou.edu

\section{ARTICLE INFO}

Article history

Received: August 28, 2018

Accepted: October 16, 2018

Published: October 31, 2018

Volume: 6 Issue: 4

Conflicts of interest: None

Funding: None

\begin{abstract}
Background of Study: Observations of limb to limb differences (bilateral asymmetry) in leg strength, power, peak oxygen uptake $\left(\mathrm{VO}_{2}\right)$ and bone mineral density has been reported in individuals with Multiple Sclerosis (MS). Objetives: The purpose of this study was to quantify the magnitude of bilateral asymmetries in oxygen uptake $\left(\mathrm{VO}_{2}\right)$ kinetics response to single leg cycling (SLC) in relapsing-remitting multiple sclerosis (MS) patients. Methods: Five MS patients ( 2 men, 3 women; age $43 \pm 7$ yrs) performed constant work rate SLC trials to determine $\mathrm{VO}_{2}$ kinetics in each leg. Asymmetry scores were used to quantify the magnitude of the bilateral asymmetries. Results: Significant asymmetries were seen in $\mathrm{VO}_{2 \text { peak }}$ and parameters of $\mathrm{VO}_{2}$ kinetics. $\mathrm{VO}_{2 \text { peak }}$ asymmetry score was significantly different than $0 \%(\mathrm{p}=0.015)$. Similarly, significant asymmetry for $\mathrm{VO}_{2}$ kinetic response to exercise as mean response time was observed $(\mathrm{p}=0.03)$. In addition the $\mathrm{VO}_{2}$ response to exercise resulted in a significant asymmetry in $\mathrm{VO}_{2}$ deficit between legs $(\mathrm{p}=0.03)$. No correlation between EDSS scores and any asymmetry scores existed. Conclusions: These findings provide insight into the potential differences in metabolic perturbation and limb specific symptomatic fatigue within the MS population.
\end{abstract}

Key words: Multiple Sclerosis, Exercise, Oxygen, Asymmetry

\section{INTRODUCTION}

Pulmonary oxygen uptake $\left(\mathrm{VO}_{2}\right)$ kinetics is generally described as the time where oxygen consumption $\left(\mathrm{VO}_{2}\right)$ is elevated to meet the demands of the cardiovascular, pulmonary, and muscular systems until the attainment of steady state $\mathrm{VO}_{2}$ during the beginning of moderate-intensity, aerobic exercise (Poole et al., 2012). Typically $\mathrm{VO}_{2}$ kinetics occurs within the first 2-3 minutes of aerobic exercise where energy production (ATP) meets energy demands. Prior to reaching steady state, phosphocreatine (PCr) and anaerobic glycolysis compensate for the energy needs (oxygen deficit) created by the delay in skeletal muscle mitochondria production of ATP (energy) until the occurrence of aerobic metabolism (Whipp et al., 1982). A clinical population of interest to examine pulmonary $\mathrm{VO}_{2}$ kinetics is multiple sclerosis (MS). MS is a chronic, autoimmune disease of the central nervous system (CNS) characterized by the destruction of the myelin that surrounds and insulates nerve fibers. This demyelination results in scarring which slows nerve signal transmission from the brain to the working muscles, resulting in neural and muscular impairments. These impairments can have a negative impact on exercise tolerance and on quality of life (Compston et al., 2008). To date, only a few researchers have studied pulmonary $\mathrm{VO}_{2}$ kinetics in individuals with $\mathrm{MS}$ and observed that the individuals with MS have significantly slower oxygen uptake at the onset of exercise than healthy individuals (Hansen et al., 2013; Ponichtera-Mulcare et al., 1993). These researchers suggest that slowed pulmonary $\mathrm{VO}_{2}$ kinetics might play a role in exercise intolerance. One interesting aspect of MS is that it can affect one side of the body more than the other (bilateral asymmetry) which has the potential to create an imbalance in function and performance. Bilateral asymmetry also contributes to fatigue, reported to be the most problematic symptom of MS, leading to reductions in strength and impairments in mobility (Ponichtera-Mulcare, 1993). Although there has been little research quantifying bilateral asymmetries in skeletal muscle function and performance in individuals with MS, the research that has been done has observed significant differences in aerobic capacity, balance, and muscular differenc- 
es between the lower limbs in a MS group compared to a matched control group by approximately $30 \%$ (Larson et al., 2013). The degree to which pulmonary $\mathrm{VO}_{2}$ kinetics affects those with MS has not been well observed. Hansen et al. (2014) observed that $\mathrm{pVO}_{2}$ kinetics was slowed in the MS population by approximately $30 \%$ of the mean response time (MRT) during double leg cycling when compared to healthy controls (Hansen et al., 2014). However, pulmonary $\mathrm{VO}_{2}$ kinetics for a single limb of an MS individual has yet to be studied. If a limb is unable to produce the required force as well as incapable of adequately delivering oxygen quickly enough to the exercising muscle, an imbalance could reduce the body's ability to metabolically synchronize the legs, compromising bilateral movements (Sandroff et al., 2013). Since fatigue is reported in the multiple sclerosis (MS) population and is a primary disabling symptom (Romani et al., 2004) and MS bilateral asymmetries in muscular strength are associated with fatigue and exercise performance, highlighting the importance of exercise related asymmetries in this population (Chung et al., 2008). The rate of increase of pulmonary oxygen uptake $\left(\mathrm{VO}_{2}\right)$ at the transition from rest to exercise depends on the coordinated muscular and cardiorespiratory responses to meet the increased $\mathrm{VO}_{2}$ demand of the activity. If this rate of change in $\mathrm{VO}_{2}$ (i.e. $\mathrm{VO}_{2}$ kinetics) at exercise onset is decreased a greater $\mathrm{VO}_{2}$ deficit and metabolic perturbation will occur, which is associated with the onset of fatigue and exercise intolerance (Poole et al., 2008). Here, we provide a pilot investigation of the single-leg $\mathrm{VO}_{2}$ kinetic response and bilateral asymmetries in a group of $\mathrm{MS}$ patients. We hypothesized that asymmetries in would exist in our sample of MS patients.

\section{METHODS}

\section{Subjects and Design}

This pilot study included 5 relapsing-remitting MS patients (2 men, 3 women; age $43 \pm 7$ yrs; stature $170.5 \pm 7.4 \mathrm{~cm}$; weight $90.2 \pm 18.5 \mathrm{~kg})$. The inclusion criteria for patients included age (18-65 years), relapsing-remitting progression, stable for at least 3 months, and Expanded Disability Statistics Scale (EDSS) of 6.0 or less. Exclusion criteria were past or present prednisone medication, past or present lower-body orthopedic injury, and history of cardiovascular disease. Informed consent was obtained per the University of Oklahoma Institutional Review Board for Research Involving Human Subjects requirements.

\section{Protocols}

A single-leg ramp exercise test $(15 \mathrm{~W} / \mathrm{min})$ to exhaustion was performed on a cycle ergometer to evaluate each participant's leg specific peak $\mathrm{VO}_{2}\left(\mathrm{VO}_{2 \text { peak }}\right)$ and gas exchange threshold (GET). Each leg was tested on a separate day with $48 \mathrm{hrs}$ between days. Following the ramp tests, over a period of 2-4 weeks, all participants performed 5-7 moderate-intensity constant work rate exercise tests in each leg corresponding to the leg specific $90 \%$ GET. These tests were performed over a minimum of 4 visits with 48 hrs between visits. At each visit at least 3 individual constant work rate tests were performed with 10-minutes between tests. Metabolic and ventilatory data were measured via a gas exchange system (True One 2400, Parvo Medics, Sandy, UT).

\section{Data Analysis and Statistics}

For modeling the $\mathrm{VO}_{2}$ response the 5-second averaged $\mathrm{VO}_{2}$ from each constant work rate test were time aligned and ensemble-averaged to provide a single response for each participant's individual leg. The mean response time (MRT) of the overall $\mathrm{VO}_{2}$ response in the transition from rest to end exercise was fit with a monoexponential model after removal of the cardiodynamic phase I (Whipp et al., 1982). The $\mathrm{VO}_{2}$ deficit was calculated as MRT $\times$ the change in $\mathrm{VO}_{2}$ from baseline. The functional "gain" of the $\mathrm{VO}_{2}$ response was calculated as the change in $\mathrm{VO}_{2}$ from baseline divided by the change in work rate. An absolute asymmetry (Chung et al., 2008) was calculated for each variable as

$$
\text { asymmetry score }=1-\left(\frac{\text { low performanceleg }}{\text { high performanceleg }}\right) 100
$$

Where the ratio was the value for the lesser performing leg divide by the value for the better performing leg for each tested variable. A value of $0 \%$ indicated and $100 \%$ indicated maximal asymmetry between legs. A one sample t-test was used to determine if the asymmetry scores were significantly different than $0 \%$ (i.e., determine if asymmetry in the limbs existed) for each tested variable (SigmaPlot/SigmaStat 12.5, Systat Software, Point Richmond, CA). Linear correlation analyses were used to determine the association between asymmetry scores and EDSS values. Statistical significance was declared when $\mathrm{P}<0.05$ (mean $\pm \mathrm{SD}$ ).

\section{RESULTS}

Patients had a mean Expanded Disability Status Scale (EDSS) score of $3.0 \pm 1.0$ (range: $2-4.5$ ) indicating mild to moderate impairment. On average our sample performed 24.0 $20.12 \mathrm{~min}-$ utes of physical activity for $4.0 \pm 2.45$ days per week. The $\mathrm{VO}_{2 \text { peak }}$, GET, MRT, SEE for the modeling, and $\mathrm{O}_{2}$ deficit for each patient's left and right legs along with individual asymmetry scores are displayed in Table 1 . The asymmetry score for $\mathrm{VO}_{2 \text { peak }}$ was significantly different than $0 \%(\mathrm{p}=0.015)$. Similarly, significant asymmetry for $\mathrm{VO}_{2}$ kinetic response to exercise, described as the MRT, was observed $(p=0.03)$. This difference in $\mathrm{VO}_{2}$, response to exercise resulted in a significant asymmetry in $\mathrm{VO}_{2}$ deficit between legs $(\mathrm{p}=0.03)$.

A representative subject's $\mathrm{VO}_{2}$ kinetic response is illustrated in Figure 1. Note the different MRTs between legs and the associated differences in $\mathrm{VO}_{2}$ deficit. There was no correlation between EDSS scores and any asymmetry scores.

\section{DISCUSSION}

This pilot study suggests that significant asymmetries in the $\mathrm{VO}_{2}$ response at exercise onset was present in relapsing-remitting MS patients. These findings support our hypothesis and provide insight into the potential differences in the met- 
Table 1. Individual data during single leg cycling

\begin{tabular}{|c|c|c|c|c|c|c|}
\hline Patient \# & Leg & $\mathrm{VO}_{2 \text { peak }}, \mathrm{ml} \mathrm{kg}^{-1} \mathrm{~min}^{-1}$ & GET, W & MRT, s & SEE & $\mathrm{O}_{2}$ deficit, $\mathrm{ml}$ \\
\hline \multirow[t]{3}{*}{1} & Right & 12.1 & 58 & 78.3 & 0.042 & 50.3 \\
\hline & Left & 12.6 & 63 & 76.3 & 0.053 & 52.4 \\
\hline & Asymmetry score & 3.97 & 7.94 & 2.63 & & 4.2 \\
\hline \multirow[t]{3}{*}{2} & Right & 21.2 & 67 & 40.4 & 0.072 & 27.2 \\
\hline & Left & 22.1 & 66 & 48.8 & 0.077 & 30.7 \\
\hline & Asymmetry score & 4.07 & 1.49 & 20.7 & & 11.3 \\
\hline \multirow[t]{3}{*}{3} & Right & 23.2 & 70 & 51.9 & 0.049 & 38.6 \\
\hline & Left & 21.3 & 64 & 37.7 & 0.041 & 23.8 \\
\hline & Asymmetry score & 8.19 & 8.57 & 37.7 & & 62.3 \\
\hline \multirow[t]{3}{*}{4} & Right & 14.1 & 43 & 36.2 & 0.048 & 13.4 \\
\hline & Left & 13.9 & 43 & 44.9 & 0.056 & 17.5 \\
\hline & Asymmetry score & 1.44 & 0.00 & 23.9 & & 23.4 \\
\hline \multirow[t]{3}{*}{5} & Right & 30.1 & 55 & 76.2 & 0.104 & 53.6 \\
\hline & Left & 27.9 & 53 & 74.2 & 0.039 & 39.0 \\
\hline & Asymmetry score & 7.31 & 3.64 & 2.6 & & 27.2 \\
\hline
\end{tabular}

Abbreviations and symbols are as follows: $\mathrm{VO}_{2 \text { peak }}$, peak oxygen uptake; GET, gas exchange threshold; MRT, mean response time; SEE, standard error of the estimate.

abolic perturbation and limb specific symptomatic fatigue previously reported in this population (Romani et al., 2008; Larson et al., 2013). Hansen et al. (2013) previously evaluated $\mathrm{VO}_{2}$ kinetics during bipedal cycling in MS patients with an EDSS score of $\sim 3.1$ and demonstrated that MS patients have a significantly slower MRT compared to healthy participants (Hansen et al., 2013). Our work expands on this data by demonstrating differences in MRT between legs of a given MS patient. The primary component of the $\mathrm{VO}_{2} \mathrm{ki}-$ netics provides an accurate analog of muscle $\mathrm{VO}_{2}$ and therefore provides unique insight into muscle energetics beyond that obtained with traditional measurements of aerobic exercise capacity (Poole et al., 2008; Poole et al., 1991). The speed of $\mathrm{VO}_{2}$ kinetics can be $\mathrm{VO}_{2}$ delivery independent and dependent, with the latter occurring in situations in which muscle $\mathrm{O}_{2}$ availability are compromised. Given the high ratio of cardiac capacity to skeletal muscle recruitment during single leg cycling it is likely that the asymmetries in $\mathrm{VO}_{2}$ kinetics are due to difference within the muscle, not bulk $\mathrm{O}_{2}$ transport. Given the MS is primarily a neurodegenerative disorder it is possible that these asymmetries are a secondary consequence of the disease and dependent on neurodegenerative dependent differences in limb training status. However, mitochondrial myopathy has been reported in some MS patients (Mao et al., 2010). It has been shown that individuals with MS exhibit bilateral asymmetry and elevated muscle fatigue, especially in the lower limbs compared to healthy controls. This study focused on possible lower leg asymmetry in $\mathrm{VO}_{2}$ kinetics as a potential mechanism for muscle fatigue in individuals with MS. A majority of MS studies on muscle function and fatigue do not specify the leg used during the study or conduct experimentation on the same leg for each participant, whether or not this leg is the weaker or stronger leg. This study utilized assessment of both limbs and calculating an asymmetry score.

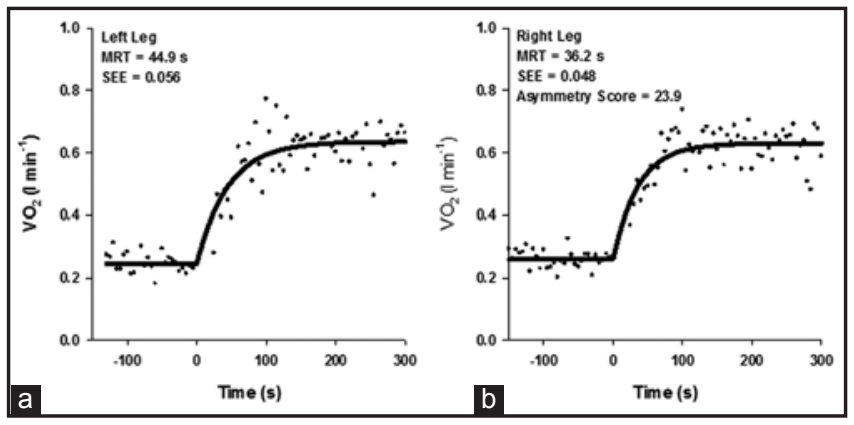

Figure 1. Representative subject's $\mathrm{O}_{2}$ kinetic response

This is one of the first studies of its kind that Should be accessed and quantified pulmonary $\mathrm{VO}_{2}$ kinetics during single leg cycling in individuals with MS. A similar study by Hansen et al. (2013) quantified pulmonary $\mathrm{VO}_{2}$ kinetics during double leg cycling in individuals with MS (Hansen et al., 2013). Due to the effect of MS on bilateral symmetry in the lower limbs in individuals with MS, it is important to access pulmonary $\mathrm{VO}_{2}$ kinetics of each limb independently to eliminate any compensation from the other leg in exercise performance. This study accurately quantified pulmonary $\mathrm{VO}_{2}$ kinetics by having each subject perform at least 5 pulmonary $\mathrm{VO}_{2}$ kinetics trials on each leg at a submaximal workload taken from 90\% of GET (Poole et al., 2012). Hansen et al. (2013) had participants perform only two pulmonary $\mathrm{VO}_{2}$ kinetics trials at $25 \%$ peak power output (Hansen et al., 2013). The present study corrected for the age of each subject by eliminating phase I from pulmonary $\mathrm{VO}_{2}$ kinetics analysis, which Hansen et al. (2013) did not take into consideration. Therefore, this study is the first stepping stone in developing proper exercise prescriptions for individuals with MS to help them to prosper in everyday life. This study is limited by the small sample size, and no control group. Likewise, no evaluation of the mechanistic determinates of 
the $\mathrm{VO}_{2}$ kinetic response occurred, which limits our interpretation of the data. However, the data reinforces the potential cardiorespiratory changes in the MS population that by association may contribute to symptoms of fatigue and exercise intolerance.

\section{CONCLUSION}

The findings from this study of significant asymmetries in $\mathrm{VO}_{2}$ peak and parameters of $\mathrm{VO}_{2}$ kinetics provide valuable insight into the potential metabolic perturbations associated with asymmetries in individuals with MS. However, further research is needed due to the small sample size and lack of control group.

\section{ACKNOWLEDGMENTS}

No external funding was used to support the current study. The authors have no relationships with any companies who will benefit from the results of the present study. In addition there was no conflict of interest with this project or manuscript.

\section{REFERENCES}

Abbiss, C. R., \& Laursen, P. B. (2005). Models to explain fatigue during prolonged endurance cycling. Sports Medicine, 35(10), 865-898.

Compston, A., \& Coles, A. (2008). Multiple Sclerosis. Multiple Sclerosis, 372(9648), 1502-17.

Chung, L.H., Remelius, J.G., Van Emmerik, R.E. \& KentBraun, J.A. (2008). Leg power asymmetry and postural control in women with multiple sclerosis. Medicine and science in sports and exercise, 40(10), 1717-24.

Hansen, D., Wens, I., Kosten, L., Verboven, K. \& Eijnde, B.O. (2013). Slowed exercise-onset Vo2 kinetics during submaximal endurance exercise in subjects with multiple sclerosis. Neurorehabilitation and neural repair, 27(1), 87-95.

Larson, R.D., McCully, K.K., Larson, D.J., Pryor, W.M. \& White LJ. (2013). Bilateral differences in lower-limb performance in individuals with multiple sclerosis. Journal of rehabilitation research and development, 50(2), 215-22.

Mao, P., \& Reddy, P.H. (2010). Is multiple sclerosis a mitochondrial disease? Biochimica et biophysica acta, 1802(1), 66-79.

Ponichtera-Mulcare, J. A. (1993). Exercise and multiple sclerosis. Medicine \& Science in Sports \& Exercise, 25(4), 451-465.

Poole, D.C., Barstow, T.J., McDonough, P., \& Jones, A.M. (2008). Control of oxygen uptake during exercise. Medicine and science in sports and exercise, 40(3), 462-74.

Poole, D. C., \& Jones, A. M. (2012). Oxygen uptake kinetics. Comprehensive Physiology, 2(2), 933-996.

Poole, D.C., Schaffartzik, W., Knight, D.R., Derion, T., Kennedy, B., Guy, H.J., Prediletto, R., \& Wagner, P.D. (1991). Contribution of exercising legs to the slow component of oxygen uptake kinetics in humans. Journal of applied physiology, 71(4), 1245-60.

Romani, A., Bergamaschi,R., Candeloro, E., Alfonsi, E., Callieco, R., \& Cosi, V. (2004). Fatigue in multiple sclerosis: multidimensional assessment and response to symptomatic treatment. Multiple sclerosis, 10(4), 462-8.

Sandroff, B. M., Sosnoff, J. J., \& Motl, R. W. (2013). Physical fitness, walking performance, and gait in multiples sclerosis. Journal of the Neurological sciences, 328(1-2), 70-76.

Whipp, B. J., Ward, S. A., Lamarra, N., Davis, J. A., \& Wasserman, K. (1982). Parameters of ventilatory and gasexchange dynamics during exercise. Journal of Applied Physiology, 52(6), 1506-1513. 